\title{
STABILITY THEORY AND ADJOINT OPERATORS FOR LINEAR DIFFERENTIAL-DIFFERENCE EQUATIONS
}

\author{
BY \\ RICHARD BELLMAN AND KENNETH L. COOKE
}

1. Introduction. In this paper, we wish to extend to linear differentialdifference equations a number of results familiar in the stability theory of ordinary linear differential equations. In this theory, one considers a system of equations of the form

$$
\frac{d x}{d t}=A(t) x, \quad x(0)=c,
$$

where $t$ is a real variable, $x$ is a column vector with $n$ rows, and $A(t)$ is an $n$-by- $n$ matrix, and a perturbed system

$$
\frac{d x}{d t}=(A(t)+B(t)) x
$$

In general terms, the stability problem is to determine conditions on the matrix $B$ sufficient to ensure that some property of all solutions of (1.1) such as boundedness or order of growth-will also be a property of all solutions of (1.2). This stability problem has been extensively investigated for ordinary differential equations, cf. Bellman, [1].

In this paper, we shall consider, instead of the functional equations of (1.1) and (1.2), the system

$$
\sum_{n=0}^{m} A_{n}(t) z^{\prime}\left(t+h_{n}\right)+\sum_{n=0}^{m} B_{n}(t) z\left(t+h_{n}\right)=0,
$$

and the perturbed system

$$
\sum_{n=0}^{m}\left\{A_{n}(t)+C_{n}(t)\right\} z^{\prime}\left(t+h_{n}\right)+\sum_{n=-1}^{m}\left\{B_{n}(t)+D_{n}(t)\right\} z\left(t+h_{n}\right)=0
$$

of linear differential-difference equations $\left({ }^{1}\right)$. Here, $A_{n}(t), B_{n}(t), C_{n}(t)$, and $D_{n}(t)$ represent given matrix functions of the real variable $t$, and $y$ represents an $n$-dimensional column vector. The "spans" $h_{0}, h_{1}, \cdots, h_{m}$ are assumed to be real, and can be supposed to satisfy the conditions $0=h_{0}<h_{1}<\cdots<h_{m}$. The stability property with which we shall he concerned is that of bounded-

Received by the editors April 12, 1958. [2].

1 For a survey of the general theory of differential-difference equations, refer to Bellman, 
ness of solutions as $t \rightarrow+\infty$. The principal results are given in Theorems 3,5 , $9,10,11$, and 12 , below.

One of the interesting features of the discussion of stability contained below is that we apparently must use the concept of the adjoint equation, rather than the more primitive concept of the inverse of a matrix which is customarily used in the stability theory for ordinary differential equations. Of fundamental importance in the latter theory is the fact that solutions of the nonhomogeneous equation

$$
\frac{d z}{d t}=A(t) z+w(t)
$$

can be represented by a simple integral operator involving $w$. In fact, if $Y(t)$ denotes the matrix solution of

$$
\frac{d Y}{d t}=A(t) Y, \quad Y(0)=I,
$$

where $I$ is the identity matrix, then

$$
z=\int_{0}^{t} Y(t) Y^{-1}(s) w(s) d s
$$

is the particular solution of (1.5) for $t>0$, satisfying the condition $z(0)=0$. The customary derivation of (1.7) utilizes the method of variation of parameters $\left({ }^{2}\right)$, and depends on an independent proof that $Y^{-1}(t)$ exists for $t \geqq 0$. Such a proof is not difficult, for differential equations $\left({ }^{3}\right)$. The simplicity of this procedure is, however, misleading since the method fails when applied to more complicated functional equations such as the nonhomogeneous counterpart of (1.3). It turns out that a more illuminating approach is furnished by use of the concept of the adjoint equation. The use of the adjoint to obtain a representation similar to (1.7) will be described in $\$ 2$ for ordinary differential equations, and in $\$ 3$ for simple differential-difference equations. In $\S 4$, we shall sketch the method for a very general system of differential-difference equations, those with "retarded argument," and in \$5-9 we shall apply the results to establish stability theorems for such systems. In later sections we shall extend the method of the adjoint equation to another broad class of differential-difference equations, those of so-called "neutral type," and we shall establish stability theorems for these equations.

The use of the adjoint in connection with differential-difference equations is apparently to be found in only one prior work, the interesting paper of N. G. deBruijn, [3]. There, however, less general classes of equations were considered, and no attention was devoted to the stability problems discussed here.

(2) Cf. [1, p. 11], for this derivation.

(3) $[1$, p. 10]. 
2. Ordinary linear differential equations. We shall attempt to find a representation, similar to (1.7), for the unique solution $z$ of the nonhomogeneous system

$$
\frac{d z}{d l}=A(t) z+w(t), \quad z(0)=0, \quad t>0,
$$

without using the inverse matrix explicitly. If we multiply (2.1) by a matrix $Y$, as yet unspecified, and integrate, we obtain the relation

$$
\int_{0}^{t} Y(s) z^{\prime}(s) d s=\int_{0}^{t} Y(s) A(s) z(s) d s+\int_{0}^{t} Y(s) w(s) d s, \quad t \geqq 0 .
$$

After an integration by parts, this takes the form

$$
Y(l) z(t)=\int_{0}^{\iota}\left\{Y^{\prime}(s)+Y(s) A(s)\right\} z(s) d s+\int_{0}^{t} Y(s) w(s) d s .
$$

In order to simplify this equation, we now ask that $Y(t)$ satisfy the equation

$$
Y^{\prime}(s)+Y(s) A(s)=0,
$$$$
0 \leqq s<t .
$$

The resulting equation for $z(t)$ can now be solved for $z(t)$. In order to avoid the use of the inverse matrix $Y(t)^{-1}$, let us impose the further condition

$$
Y(t)=I \text {. }
$$

Provided that $A(t)$ is continuous, (2.4) possesses a unique solution $Y(s)$ satisfying (2.5) and defined for $t \geqq s \geqq 0$. With this choice of $Y$, we obtain from (2.3),

$$
z(l)=\int_{0}^{t} Y(s) w(s) d s,
$$

which is the desired relation.

The system in (2.4) and the original system

$$
Y^{\prime}(t)=A(t) Y(t)
$$

are said to be adjoint $\left({ }^{4}\right)$ to one another. It is important to note that the function $Y$ defined by (2.4) and (2.5) actually depends on two variables, $s$ and $t$. In fact, it will be convenient for us to indicate this explicitly by adopting the notation $Y(s, t)$ for $Y$. The relations (2.4), (2.5), and (2.6) then take the forms

$$
\begin{aligned}
\frac{\partial}{\partial s} Y(s, t) & =-Y(s, t) A(s), \quad(t>0 ; s>0), \\
Y(t, t) & =I,
\end{aligned}
$$

(4) The equation adjoint to (2.7) is of ten defined to be $Y^{\prime}=-A * Y$ where $A^{*}$ is the conjugate transpose of $A$; see Coddington and Levinson, [4, p. 70]. 
and

$$
z(t)=\int_{0}^{t} Y(s, t) w(s) d s .
$$

It is easy to verify that if $X(t)$ is the unique solution of (1.6) satisfying $X(0)=I$, then the function

$$
Y(s, t)=X(t) X^{-1}(s)
$$

is the unique solution of (2.8) and (2.9). Therefore, (2.10) and (1.7) are equivalent results.

3. The scalar linear differential-difference equation. In order to illustrate the application of the above procedure to differential-difference equations, let us first examine the simple class of scalar equations of the form

$$
u^{\prime}(t+h)+b(t) u(t)+c(t) u(t+h)=w(t)
$$

$t>t_{0}$,

where $b(t), c(t)$, and $w(t)$ are given real scalar functions and $h$ is a given positive number.

We begin by making some observations concerning the existence and uniqueness of solutions of (3.1). An initial condition appropriate to the functional equation in (3.1) has the form

$$
u(t)=\phi(t),
$$

$$
t_{0} \leqq t \leqq t_{0}+h
$$

where $\phi(t)$ is a prescribed real function. Let us suppose that $b(t), c(t)$, and $w(t)$ are continuous for $t \geqq t_{0}$ and that $\phi(t)$ is continuous for $t_{0} \leqq t \leqq t_{0}+h$. Then from (3.1) we see that $u^{\prime}(t+h)+c(t) u(t+h)$ is determined over $t_{0}<t<t_{0}+h$, by the given values of $\phi(t)$, as a continuous function.

It follows that there is just one way to define $u(t)$ over $t_{0} \leqq t \leqq t_{0}+2 h$ so that $u(t)$ is continuous at $t_{0}+h$, equal to $\phi(t)$ for $t_{0} \leqq t \leqq t_{0}+h$, and so that the equation in (3.1) is satisfied for $t_{0}<t<t_{0}+h$. Moreover, it is clear that by repeating this argument, we can continue the function $u(t)$ from one interval of length $h$ to another, and that this continuation is made unique by the requirement that $u(t)$ be continuous. In short, there is a unique continuous solution of the equations in (3.1) and (3.2). Note that $u^{\prime}(t)$ is necessarily also continuous for $t>t_{0}+h$.

We shall now find a representation, analogous to (2.10), for the particular solution of the equation in (3.1) for which $\phi(t)$ is identically zero. Imitating the procedure of $\S 2$, we multiply the equation in (3.1) by a function $v(s, t)(5)$, as yet unspecified, and integrate with respect to $s$ from $t_{0}$ to $t$. Provided that $v(s, t)$ is differentiable with respect to $s$ for $t_{0}<s<t$, we can then integrate by parts as above, deriving in this way the relation

(5) We shall indicate the dependence of $v$ on $t$ as well as on $s$ from the start this time. 


$$
\begin{aligned}
v(t, t) u(t+h) & -\int_{t_{0}}^{t}\left\{\frac{\partial v(s, t)}{\partial s}\right\} u(s+h) d s \\
& +\int_{t_{0}}^{t} v(s, t) b(s) u(s) d s \\
& +\int_{t_{0}}^{t} v(s, t) c(s) u(s+h) d s \\
= & \int_{t_{0}}^{t} v(s, t) w(s) d s .
\end{aligned}
$$

Since $u(s)=0$ for $t_{0} \leqq s \leqq t_{0}+h$, we have

$$
\int_{t_{0}}^{t} v(s, t) b(s) u(s) d s=\int_{t_{0}}^{t-h} v(s+h, t) b(s+h) u(s+h) d s, \quad t>t_{0} .
$$

Therefore

$$
\begin{array}{ll}
v(t, t) u(t+h) & \\
\quad+\int_{t_{0}}^{t-h}\left\{-\frac{\partial}{\partial s} v(s, t)+v(s+h, t) b(s+h)+v(s, t) c(s)\right\} u(s+h) d s \\
+\int_{t-h}^{t}\left\{-\frac{\partial}{\partial s} v(s, t)+v(s, t) c(s)\right\} u(s+h) d s & t>t_{0} .
\end{array}
$$

We now ask that $v$ satisfy the adjoint equation

$$
\text { (3.6) }-\frac{\partial v}{\partial s}(s, t)+v(s+h, t) b(s+h)+v(s, t) c(s)=0, \quad t>t_{0}, t_{0}<s<t-h \text {, }
$$

as well as the relations

$$
\begin{aligned}
-\frac{\partial v}{\partial s}(s, t)+v(s, t) c(s) & =0, \quad t-h<s<t, \\
v(s, t) & =1 \text { at } s=t .
\end{aligned}
$$

With this choice of $v$, we at once obtain

$$
u(t+h)=\int_{t_{0}}^{t} v(s, t) w(s) d s, \quad t>t_{0} .
$$

The Equations (3.7) and (3.8) can be combined into the single equation

$$
v(s, t)=\exp \left[-\int_{0}^{t} c\left(s_{1}\right) d s_{1}\right], \quad t-h \leqq s \leqq t .
$$


If $b(s)$ and $c(s)$ are continuous for $s \geqq t_{0}$, we can show by the continuation method that there is a unique function $v(s, t)$, defined and continuous for $t>t_{0}, t_{0} \leqq s \leqq t$, which satisfies (3.6) and (3.10). Moreover, since $\partial v(s, t) / \partial s$ is continuous for $s<t-h$ and $t-h<s<t$, and $v(s, t)$ is continuous for $s<t$, the manipulations used above are justified, and the conclusion (3.9) is valid.

Equation (3.1) is said to be an equation with retarded argument, because the arguments of $u$ do not exceed any argument of $u^{\prime}$. This is not true of the equation

$$
u^{\prime}(t+h)+a(t) u^{\prime}(t)+b(t) u(t)+c(t) u(t+h)=w(t),
$$

which is said to be of neutral type. Equations of neutral type are somewhat more troublesome, because discontinuities in the derivative of the initial function are propagated-that is, they appear repeatedly in the derivative of the solution. For this reason, we shall defer their consideration until $\$ \$ 11-15$ below. In the next section, we shall show how to extend the method given above to the most general system of linear differential-difference equations with retarded argument. The results will be embodied there in a formal theorem.

4. The matrix equation with retarded argument. The most general linear, nonhomogeneous system of differential-difference equations can be put in the form $\left(^{6}\right)$

$$
\sum_{n=0}^{m} A_{n}(t) z^{\prime}\left(t+h_{n}\right)+\sum_{n=0}^{m} B_{n}(t) z\left(t+h_{n}\right)=w(t)
$$

where $A_{n}(t)$ and $B_{n}(t)$ are given $N$ by $N$ matrices $(n=0,1, \cdots, m)$ and $w(t)$ is a given column vector of $N$ dimensions. We shall suppose that

$$
0=h_{0}<h_{1}<\cdots<h_{m} .
$$

If $A_{m}(t)$ is nonsingular for $t>t_{0}$, whereas $A_{0}(t), \cdots, A_{m-1}(t)$ are identically zero, the system (4.1) takes the simpler form

$$
z^{\prime}\left(t+h_{m}\right)+\sum_{n=0}^{m} B_{n}(t) z\left(t+h_{n}\right)=w(t), \quad t>\iota_{0},
$$

and is said to be an equation with retarded argument. We shall now show that the method of $\$ 3$ can be applied without essential change to (4.3).

We first observe that if $B_{n}(t)(n=0,1, \cdots, m)$, and $w(t)$ are continuous for $t>t_{0}$ and if $\phi(t)$ is continuous for $t_{0} \leqq t \leqq t_{0}+h_{m}$, then there is a unique continuous solution of (4.3) and

$$
u(t)=\phi(t), \quad t_{0} \leqq t \leqq t_{0}+h_{m} .
$$

( $\left.{ }^{6}\right)$ This system includes, as a special case, the scalar equation $\sum_{k=0}^{m} \sum_{l-0}^{N} a_{k l}(t) u^{(l)}\left(t+h_{k}\right)$ $=w(t)$ which has been studied by Wright, $[7 ; 8]$, and by many other authors. To see this, make the substitution $u^{(l)}(t)=z_{l+1}(t)$ and let $z$ denote the vector whose components are $z_{1}, z_{2}, \cdots, z_{N}$. 
The proof of this statement is obtained, as before, by continuing the solution from interval to interval. In fact, from the relation

$$
z^{\prime}\left(t+h_{m}\right)+B_{m}(t) z\left(t+h_{m}\right)=w(t)-\sum_{n=0}^{m-1} B_{n}(t) z\left(t+h_{n}\right)
$$

we see that there is just one way to define $u(t)$ over $t_{0} \leqq t \leqq t_{0}+2 h_{m}-h_{m-1}$ so that (4.3) and (4.4) are satisfied and $u(t)$ is continuous at $t=t_{0}+h_{m}$. Knowing $u(t)$ over $t_{0} \leqq t \leqq t_{0}+2 h_{m}-h_{m-1}$, we can again use (4.5) to see that $u(t)$ is uniquely determined over $t_{0} \leqq t \leqq t_{0}+3 h_{m}-2 h_{m-1}$, and so on. Note also that $z^{\prime}(t)$ is continuous for $t>t_{0}+h_{m}$.

The adjoint equation and kernel $Y(s, t)$ are in this case defined as follows:

Definition. Let $Y(s, t)$ denote the unique matrix function, defined for $t>t_{0}, t_{0} \leqq s \leqq t+h_{m}$, which is continuous for $t_{0} \leqq s \leqq t$, which satisfies the initial condition

$$
Y(s, t)=\left\{\begin{array}{ll}
0, & t<s \leqq t+h_{m} \\
I, & s=t
\end{array}\right\}
$$

and the adjoint equation

$$
\begin{aligned}
-\frac{\partial}{\partial s} Y(s, t)+\sum_{n=0}^{m} Y\left(s+h_{m}-h_{n}, t\right) B_{n}\left(s+h_{m}-h_{n}\right)=0, \\
\left(t>t_{0} ; t_{0}<s<t-h_{m} \text { and } t-h_{m}<s<t\right) .
\end{aligned}
$$

By a continuation argument similar to that used above, we can show that the stated conditions actually define a unique $Y(s, t)$. It should be noted that we have defined $Y(s, t)$ not only for $t_{0} \leqq s \leqq t$, but also for $t<s \leqq t+h_{m}$, in contrast to our procedure for the scalar equation (3.1). This is actually not necessary, but serves to simplify the mechanics of the argument. For example, it makes it unnecessary to state explicitly the initial values of $Y(s, t)$ for $t-h_{m} \leqq s \leqq t$, which are more complicated than the relations (3.8) or (3.11) for the scalar equation. We also observe that $\partial Y(s, t) / \partial s$ is continuous for $t_{0}<s<t-h_{m}$ and piecewise continuous for $t-h_{m}<s<t$.

The basic theorem on the representation of solutions of (4.3) is as follows.

THEOREM 1. Suppose that $w(t)$ is a continuous vector function and $B_{n}(t) a$ continuous matrix function $(n=0,1, \cdots, m)$ for $t>t_{0}$. Let $Y(s, t)$ denote the kernel matrix defined above. Then the unique continuous solution of (4.3) for $t>t_{0}$ which satisfies the initial condition

$$
z(t)=0, \quad t_{0} \leqq t \leqq t_{0}+h_{m}
$$

is given by the formula

$$
z\left(t+h_{m}\right)=\int_{t_{0}}^{t} Y(s, t) w(s) d s, \quad\left(t>t_{0}\right) .
$$


Proof. We multiply (4.3) by $Y(s, t)$ and integrate. Since $\partial Y / \partial s$ is piecewise continuous and $Y$ is continuous, integration by parts is allowable and the result is

$$
\begin{gathered}
Y(t, t) z\left(t+h_{m}\right)-\int_{t_{0}}^{t} \frac{\partial Y}{\partial s}(s, t) z\left(s+h_{m}\right) d s+\sum_{n=0}^{m} \int_{t_{0}}^{t} Y(s, t) B_{n}(s) z\left(s+h_{n}\right) d s \\
=\int_{t_{0}}^{t} Y(s, t) w(s) d s .
\end{gathered}
$$

Since $z(s)=0$ for $t_{0} \leqq s \leqq t_{0}+h_{m}$, the following relation holds:

$$
\begin{aligned}
& \int_{t_{0}}^{t} Y(s, t) B_{n}(s) z\left(s+h_{n}\right) d s=\int_{t_{0}+h_{m}-h_{n}}^{t} Y(s, t) B_{n}(s) z\left(s+h_{n}\right) d s \\
& =\int_{t_{0}}^{t-h_{m}+h_{n}} Y\left(s+h_{m}-h_{n}, t\right) B_{n}\left(s+h_{m}-h_{n}\right) z\left(s+h_{m}\right) d s, \quad(n=0,1, \cdots, m) .
\end{aligned}
$$

From the definition in (4.6), it follows that

$$
\begin{aligned}
& \int_{t_{0}}^{t} Y(s, t) B_{n}(s) z\left(s+h_{n}\right) d s \\
& =\int_{t_{0}}^{t} Y\left(s+h_{m}-h_{n}, t\right) B_{n}\left(s+h_{m}-h_{n}\right) z\left(s+h_{m}\right) d s, \quad(n=0,1,2, \cdots, m) .
\end{aligned}
$$

Therefore,

$$
\begin{aligned}
z\left(t+h_{m}\right) & +\int_{t_{0}}^{t}\left\{-\frac{\partial Y(s, t)}{\partial s}\right. \\
& \left.+\sum_{n=0}^{m} Y\left(s+h_{m}-h_{n}, t\right) B_{n}\left(s+h_{m}-h_{n}\right)\right\} z\left(s+h_{m}\right) d s \\
& =\int_{t_{0}}^{t} Y(s, t) w(s) d s .
\end{aligned}
$$

Referring to (4.7), we obtain the required relation (4.8).

5. A stability theorem for differential equations. In order to see how the foregoing representation theorem can be applied to stability theory, we shall first consider ordinary differential equations, and shall establish the following theorem; cf. [1, p. 43].

TheOREM 2. Let $A(t)$ and $B(t)$ be continuous $\left.{ }^{7}\right)$ for $t>t_{0}$. Then a sufficient condition for all solutions of

(7) This condition can be considerably relaxed (integrability is sufficient), but it is our purpose to describe the method employed rather than to obtain the most general results possible. 


$$
\frac{d z}{d t}=(A(t)+B(t)) z
$$

to be bounded as $t \rightarrow+\infty$ is that

(i) all solutions of

$$
\frac{d y}{d t}=A(t) y
$$

be bounded as $t \rightarrow+\infty$;

$$
\begin{gathered}
\int^{\infty}\|B(t)\| d t<\infty ; \\
\|Y(s, t)\| \leqq c_{1}
\end{gathered}
$$

where $c_{1}$ is a constant, for $t \geqq 0,0 \leqq s \leqq t$. Here $Y$ denotes the kernel function defined by Equations (2.8) and (2.9), and the norms $\|B\|$ and $\|Y\|$ denote the sum of the absolute values of the elements of the matrices $B$ and $Y$ respectively.

Proof. From the discussion of $\S 2$, we know that every solution of the nonhomogeneous system (2.1), with continuous $w$, has the form

$$
z(t)=y(t)+\int_{0}^{t} Y(s, t) w(s) d s
$$

where $y(t)$ is a solution of the corresponding homogeneous system (5.2). Equation (5.1) has the same form as (2.1) if we take $w=B z$. It follows that any continuous solution of (5.1) must also satisfy the integral equation

$$
z=y+\int_{0}^{t} Y(s, t) B(s) z(s) d s
$$

for some solution $y$ of (5.2). By hypotheses (i) and (iii),

$$
\|z\| \leqq c+c_{1} \int_{0}^{t}\|B(s)\|\|z(s)\| d s .
$$

It follows from a lemma $\left.{ }^{8}\right)$ of frequent use in stability theory that

$$
\|z\| \leqq c \exp \left[c_{1} \int_{0}^{t}\|B(s)\| d s\right] .
$$

Thus, every solution of (5.1) is bounded as $t \rightarrow+\infty$.

Of particular importance is the case in which $A(t)=A$, a constant matrix. The solutions of (5.2) then have $\left({ }^{9}\right)$ the form $e^{A t} y_{0}$, where $y_{0}$ is a constant

(8) Cf. [1, p. 35].

(9) Cf. $[1$, p. 12]. 
vector and $e^{A t}$ is the exponential matrix. In this case, $Y(s, t)=e^{A t} e^{-A s}=e^{A(t-s)}$. By hypothesis (i), there is a constant $c_{1}$ such that $\left\|e^{A t}\right\| \leqq c_{1}$ for $t \geqq 0$; hence, $\|Y(s, t)\| \leqq c_{1}$ for $0 \leqq s \leqq t$. That is, if $A$ is constant, the hypothesis (iii) is superfluous. This is a well-known result $\left({ }^{10}\right)$.

6. A stability theorem for equations with retarded argument. We shall now prove similar results for systems of linear differential-difference equations with retarded argument.

TheOREM 3. Let $B_{n}(t)$ and $D_{n}(t)$ be continuous for $t>t_{0}(n=0,1, \cdots, m)$. Then a sufficient condition for all continuous solutions of

$$
z^{\prime}\left(t+h_{m}\right)+\sum_{n=0}^{m}\left(B_{n}(t)+D_{n}(t)\right) z\left(t+h_{n}\right)=0, \quad t>t_{0},
$$

to be bounded as $t \rightarrow+\infty$ is that

(i) all continuous solutions of the unperturbed equation

$$
y^{\prime}\left(t+h_{m}\right)+\sum_{n=0}^{m} B_{n}(t) y\left(t+h_{n}\right)=0
$$

be bounded as $t \rightarrow+\infty$;

$$
\int^{\infty}\left\|D_{n}(t)\right\| d t<\infty, \quad n=0,1,2, \cdots, m,
$$

where $c_{1}$ is a constant, for $t \geqq t_{0}, t_{0} \leqq s \leqq t$. Here $Y(s, t)$ denotes the kernel function defined in $\$ 4$.

Proof. From Theorem 1, we know that every continuous solution of the nonhomogeneous system (4.3), with continuous $w$, has the form

$$
z\left(t+h_{m}\right)=y\left(t+h_{m}\right)+\int_{t_{0}}^{t} Y(s, t) w(s) d s
$$

where $y(t)$ is a solution of the corresponding homogeneous system (6.2). Equation (6.1) has the form of (4.3) where

$$
w(t)=-\sum_{n=0}^{m} D_{n}(t) z\left(t+h_{n}\right) .
$$

For a continuous solution $z$ of (6.1), this $w$ is continuous, and therefore

$$
z\left(t+h_{m}\right)=y\left(t+h_{m}\right)-\sum_{n=0}^{m} \int_{t_{0}}^{t} Y(s, t) D_{n}(s) z\left(s+h_{n}\right) d s, \quad t>t_{0} .
$$

(10) Cf. $[1$, p. 34]. 
From this integral equation, we can deduce the boundedness of $z$. Using hypotheses (i) and (iii), we have

$$
\begin{aligned}
\left\|z\left(t+h_{m}\right)\right\| & \leqq c+c_{1} \sum_{n=0}^{m} \int_{t_{0}}^{t}\left\|D_{n}(s)\right\|\left\|z\left(s+h_{n}\right)\right\| d s \\
& \leqq c+c_{1} \sum_{n=0}^{m} \int_{t_{0}}^{t+h_{n}-h_{m}}\left\|D_{n}\left(s+h_{m}-h_{n}\right)\right\| \cdot\left\|z\left(s+h_{m}\right)\right\| d s .
\end{aligned}
$$

It follows, using the lemma to which we referred above, that

$$
\left\|z\left(t+h_{m}\right)\right\| \leqq c \exp \left[c_{1} \sum_{n=0}^{m} \int_{t_{0}}^{t+h_{n}-h_{m}}\left\|D_{n}\left(s+h_{m}-h_{n}\right)\right\| d s\right]
$$

and hence, by (ii), that $z$ is bounded as $t \rightarrow+\infty$.

7. Equations with constant coefficients. Again it is important to examine the special case of constant coefficients in (6.2). Suppose that $B_{n}(t)=B_{n}$, $(n=0,1, \cdots, m)$, where $B_{n}$ is constant. We see from the adjoint equation (4.7) that $Y(s, t)$ can be continued indefinitely in the negative $s$ direction, and hence can be regarded as defined and continuous for $-\infty<s \leqq t$. By making the substitutions

$$
\begin{aligned}
\tau & =t-s+t_{0}+h_{m}, \\
X(\tau, t) & =Y(s, t),
\end{aligned}
$$

we can bring the adjoint equation into a simpler form. In fact, we see that $X(\tau, t)$ is defined for $t>t_{0}, t_{0} \leqq \tau<+\infty$, and that $X(\tau, t)$ is continuous for $t_{0}+h_{m} \leqq \tau<+\infty$. Furthermore, $X$ satisfies the initial conditions

$$
\begin{array}{rlrl}
X(\tau, t) & =0, & t_{0} & \leqq \tau<t_{0}+h_{m}, \\
& =I, \quad \tau=t_{0}+h_{m},
\end{array}
$$

and the differential-difference equation

$$
\begin{aligned}
& \frac{\partial X}{\partial \tau}(\tau, l)+\sum_{n=0}^{m} X\left(\tau-h_{m}+h_{n}, t\right) B_{n}=0, \\
& \quad t>t_{0}, t_{0}+h_{m}<\tau<t_{0}+2 h_{m} \text { and } t_{0}+2 h_{m}<\tau<\infty .
\end{aligned}
$$

From (7.2) and (7.3), moreover, it is clear that $X(\tau, t)$ is actually independent of $t$; we shall henceforth write $X(\tau)$ rather than $X(\tau, t)$. It is now clear that condition (iii) of Theorem 3 can be replaced by the requirement that $\|X(\tau)\|$ $\leqq c_{1}$ for $t_{0}+h_{m} \leqq \tau \leqq t+h_{m}$ or simply for $\tau \geqq t_{0}$. Hence we have proved

THEOREM 4. Let $D_{n}(t)$ be continuous for $t>t_{0}(n=0,1, \cdots, m)$. Then $a$ sufficient condition in order that all continuous solutions of

$$
z^{\prime}\left(t+h_{m}\right)+\sum_{n=0}^{m}\left(B_{n}+D_{n}(t)\right) z\left(t+h_{n}\right)=0
$$


be bounded as $t \rightarrow+\infty$ is that

(i) all continuous solutions of

$$
y^{\prime}\left(t+h_{m}\right)+\sum_{n=0}^{m} B_{n} y\left(t+h_{n}\right)=0
$$

be bounded as $t \rightarrow+\infty$;

$$
\int^{\infty}\left\|D_{n}(t)\right\| d t<\infty, \quad n=0,1, \cdots, m ;
$$

(iii) the unique solution of

$$
X(t)=\left\{\begin{array}{ll}
0, & t_{0} \leqq t<t_{0}+h_{m} \\
I, & t=t_{0}+h_{m}
\end{array}\right\}
$$

and

$$
X^{\prime}\left(t+h_{m}\right)+\sum_{n=0}^{m} X\left(t+h_{n}\right) B_{n}=0, \quad t_{0}<t<t_{0}+h_{m} \text { and } t_{0}+h_{m}<t
$$

which is continuous for $t \geqq t_{0}+h_{m}$, be bounded as $t \rightarrow+\infty$.

In (7.6) and (7.7), we have written $t$ in place of $\tau$.

8. A lemma. If each pair of matrices $B_{k}$ and $B_{l}, k, l=0,1, \cdots, m$, commutes, Theorem 4 can be replaced by a simpler theorem in which hypothesis (iii) is suppressed, as was true for systems of ordinary differential equations. We shall show this with the aid of the following lemma.

Lemma. Let $X(t)$ be the unique solution of (7.6) and (7.7) which is continuous for $t \geqq t_{0}+h_{m}$. Suppose that

$$
B_{k} B_{l}=B_{l} B_{h}, \quad k, l=0,1, \cdots, m .
$$

Then $X(t)$ as defined by (7.7) satisfies the equation

$$
X^{\prime}\left(t+h_{m}\right)+\sum_{n=0}^{m} B_{n} X\left(t+h_{n}\right)=0, \quad\left(t_{0}<t<t_{0}+h_{m} \text { and } t>t_{0}+h_{m}\right) .
$$

Proof. Let $X_{1}$ and $X_{2}$ be defined as follows:

$$
\begin{aligned}
& X_{1}\left(t+h_{m}\right)=\sum_{n=0}^{m} X\left(t+h_{n}\right) B_{n}, \\
& X_{2}\left(t+h_{m}\right)=\sum_{n=0}^{m} B_{n} X\left(t+h_{n}\right),
\end{aligned}
$$

Then 


$$
\begin{aligned}
X_{1}^{\prime}\left(t+h_{m}\right) & =\sum_{n=0}^{m} X^{\prime}\left(t+h_{n}\right) B_{n} \\
& =-\sum_{n=0}^{m}\left(\sum_{k=0}^{m} X\left(t+h_{n}+h_{k}-h_{m}\right) B_{k}\right) B_{n} \\
& =-\sum_{n=0}^{m} X_{1}\left(t+h_{n}\right) B_{n}
\end{aligned}
$$

for $t>t_{0}$, except at certain isolated points in the interval $t_{0}<t \leqq t_{0}+h_{m}$. Also, in similar fashion,

$$
X_{2}^{\prime}\left(t+h_{m}\right)=-\sum_{n=0}^{m} X_{2}\left(t+h_{n}\right) B_{n} .
$$

'Thus $X_{1}$ and $X_{2}$ are both solutions of (7.7) for $t>t_{0}$, continuous for $t \geqq t_{0}+h_{m}$. If it can be shown that they are identical for $t_{0}<t<t_{0}+h_{m}$, it will follow from the uniqueness of continuous solutions of (7.7) that they are identical for $t>t_{0}$, and (8.1) will then be an immediate consequence of (7.7).

That $X_{1}$ and $X_{2}$ are equal for $t_{0}<t<t_{0}+h_{m}$ can be shown by showing that $X\left(t+h_{n}\right)$ commutes with $B_{n}$ for $n=0,1, \cdots, m ; t_{0}<t<t_{0}+h_{m}$. We shall sketch a proof of this by direct calculation. From (7.7) and (7.6)

$$
X^{\prime}\left(t+h_{m}\right)+X\left(t+h_{m}\right) B_{m}=0, \quad t_{0}<t<t_{0}+h_{m}-h_{m-1} .
$$

Hence

$$
X\left(t+h_{m}\right)=e^{B_{m} t}, \quad t_{0}<t<t_{0}+h_{m}-h_{m-1} .
$$

Since $B_{n}$ commutes with $B_{m}$, and hence also $e^{B_{m} t}$, it follows that $X\left(t+h_{m}\right)$ commutes with any $B_{n}$ for $t_{r} \leqq t \leqq t_{0}+h_{m}-h_{m-1}$. Next,

$$
\begin{aligned}
X^{\prime}\left(t+h_{m}\right)+X\left(t+h_{m}\right) & B_{m}+X\left(t+h_{m-1}\right) B_{m-1}=0, \\
& \left(t_{0}+h_{m}-h_{m-1}<t<t_{0}+h_{m}-h_{m-2}\right),
\end{aligned}
$$

and

$$
\begin{aligned}
X^{\prime}\left(t+h_{m}\right)+X\left(t+h_{m}\right) B_{m} & =-e^{B_{m}\left(t+h_{m-1}-h_{m}\right)} B_{m-1}, \\
& \left(t_{0}+h_{m}-h_{m-1}<t<t_{0}+2 h_{m}-2 h_{m-1}\right) .
\end{aligned}
$$

The solution of this differential equation is readily found to be of the form

$$
X\left(t+h_{m}\right)=e^{-B_{m} t} C-\int_{t_{0}}^{t} e^{B_{m}\left(s+h_{m-1}-h_{m}\right)} B_{m-1} e^{-B_{m}(t-s)} d s
$$

where $C$ is chosen to yield continuity of $X\left(t+h_{m}\right)$ at $t=t_{0}+h_{m}-h_{m-1}$. Since $B_{n}$ commutes with $B_{m}$ and $B_{m-1}$, and hence also $e^{B_{m} \varepsilon}$, it follows that $X\left(t+h_{m}\right)$ commutes with any $B_{n}$ for $t_{0}+h_{m}-h_{m-1} \leqq t \leqq t_{0}+2 h_{m}-2 h_{m-1}$. Continuing in this way, we find that $X\left(t+h_{m}\right)$ is determined over $t_{0} \leqq t \leqq t_{0}+h_{m}$ as a com- 
bination of $B_{m}, \cdots, B_{0}$ and exponentials $e^{B_{m} t}$, etc., and consequently that $X(t)$ commutes with every $B_{n}$ for $t_{0} \leqq t \leqq t_{0}+2 h_{m}$. This implies that $X_{1}\left(t+h_{m}\right)$ $=X_{2}\left(t+h_{m}\right)$ for $t_{0}<t<t_{0}+h_{m}$, and completes the proof of the lemma.

9. A stability theorem for equations with constant coefficients. Let us now prove the following theorem.

Theorem 5. Let $D_{n}(t)$ be continuous for $t>t_{0},(n=0,1, \cdots, m)$. Suppose that $B_{k} B_{l}=B_{l} B_{k}(k=0,1, \cdots, m ; l=0,1, \cdots, m)$. Then a sufficient condition in order that all continuous solutions of

$$
z^{\prime}\left(t+h_{m}\right)+\sum_{n=0}^{m}\left(B_{n}+D_{n}(t)\right) z\left(t+h_{n}\right)=0,
$$

be bounded as $t \rightarrow+\infty$ is that

(i) all solutions of

$$
y^{\prime}\left(t+h_{m}\right)+\sum_{n=0}^{m} B_{n} y\left(t+h_{n}\right)=0
$$

continuous for $t \geqq t_{0}+h_{m}$, be bounded as $t \rightarrow+\infty$;

(ii) $\int \infty\left\|D_{n}(t)\right\| d t<\infty$.

Proof. We shall show that the commutativity $B_{l} B_{k}=B_{k} B_{l}$ enables us to deduce condition (iii) of Theorem 4 from condition (i). If $X(t)$ is any solution of (7.7), continuous for $t \geqq t_{0}+h_{m}$, and if $c$ is a constant vector, then $X(t) c$ is a vector solution of (9.2), continuous for $t \geqq t_{0}+h_{m}$. By hypothesis (i), therefore, $X(t)$ must be bounded as $t \rightarrow+\infty$. In particular, the unique solution $X(t)$ of (7.6) and (7.7) must be bounded as $t \rightarrow+\infty$.

10. Boundedness of solutions of the unperturbed system. It is well-known that a necessary and sufficient condition for the boundedness of all solutions of the unperturbed system of differential equations

$$
\frac{d y}{d t}=A y
$$

is that the roots of the associated algebraic equation

$$
\operatorname{det}(A-s I)=0
$$

either have negative real parts, or have zero real parts and be "of simple type" $\left.{ }^{11}\right)$. Theorem 2 can therefore be given an alternative form in which hypothesis (i) is replaced by the above assertion concerning the roots of (10.2).

In the same way, hypothesis (i) of Theorem 5 can be replaced by an assertion concerning the roots of the transcendental equation

(11) Cf. Bellman, [1, pp. 19-25]. Multiple roots on the imaginary axis may or may not give rise to unbounded solutions. 


$$
\operatorname{det}\left(s e^{s h_{m}} I+\sum_{n=0}^{m} B_{n} e^{s h_{n}}\right)=0 .
$$

Here, however, the problem is considerably more complicated, since the equation in (10.3) has infinitely many roots. One of the most expeditious methods of treating this problem is the method of the Laplace transform. Since any solution of the equation in (9.2) is, with its first derivative, of exponential order $\left({ }^{12}\right)$ as $t \rightarrow+\infty$, it follows from (9.2) that

$$
\int_{t_{0}}^{\infty} y^{\prime}\left(t+h_{m}\right) e^{-s t} d t+\sum_{n=0}^{m} B_{n} \int_{t_{0}}^{\infty} y\left(t+h_{n}\right) e^{-s t} d t=0 .
$$

By integrating by parts and making simple transformations, we find $\left({ }^{12}\right)$ that

$$
T(s) \int_{t_{0}}^{\infty} y(t) e^{-s t} d t=y^{*}(s)
$$

where

$$
\begin{aligned}
T(s)= & s e^{s h_{m}} I+\sum_{n=0}^{m} B_{n} e^{s h_{n}} \\
y^{*}(s)= & y\left(t_{0}+h_{m}\right) e^{-s t_{0}}+s e^{s h_{m}} \int_{t_{0}}^{t_{0}+h_{m}} y(t) e^{-s t} d t \\
& +\sum_{n=0}^{m} B_{n} e^{s h_{n}} \int_{t_{0}}^{t_{0}+h_{n}} y(t) e^{-s t} d t .
\end{aligned}
$$

Provided the roots of (10.3) all have nonpositive real parts, the matrix $T(s)$ is nonsingular for $\operatorname{Re} s>0$, and we have

$$
\int_{t_{0}}^{\infty} y(t) e^{-s t} d t=T^{-1}(s) y^{*}(s), \quad \operatorname{Re} s>0 .
$$

Furthermore, we can suppose $\left({ }^{13}\right)$ that $y(t)$ and $y^{\prime}(t)$ are continuous for $t>t_{0}$, and hence that $y(t)$ is of bounded variation in any finite interval in which $t>t_{0}$. A standard inversion theorem for the Laplace transform can therefore be applied. We have

$$
y(t)=\frac{1}{2 \pi i} \lim _{t \rightarrow+\infty} \int_{u-i v}^{u+i v} e^{t s} T^{-1}(s)\left(y^{*}\right)(s) d s, \quad\left(t>t_{0}\right),
$$

where $u$ is any sufficiently large positive number.

(12) Cf. Wright, [6].

(18) In the existence-uniqueness theorem of $\S 5$, we supposed only that $y(t)$ is continuous in the initial interval $t_{0} \leqq t \leqq t_{0}+h_{m}$, but as shown there it follows that $y^{\prime}(t)$ is continuous for $t>t_{0}+h_{m}$. There is no loss of generality in supposing that $y^{\prime}(t)$ is continuous for $t>t_{0}$, since if not we would use $t_{0}+h_{m}$ as lower limit in the above integrations. 
From Equation (10.8), we can determine the asymptotic behavior of $y(t)$, and in particular can deduce conditions for the boundedness of $y(t)$ as $t \rightarrow+\infty$. We have assumed that all roots of (10.3) have nonpositive real parts. It follows from results of Langer, [5], and Wright, [7], that there are at most a finite number of roots with zero real parts, and that all other roots are bounded away from the imaginary axis. We can therefore shift the integration contour in (10.8) to the left of the imaginary axis, obtaining

$$
\begin{aligned}
y(t)= & \sum \text { Residue of }\left\{e^{t_{s}} T^{-1}(s) y^{*}(s)\right\} \text { at } s_{r} \\
& +\frac{1}{2 \pi i} \lim _{v \rightarrow+\infty} \int_{u-i v}^{u+i v} e^{t_{s}} T^{-1}(s) y^{*}(s) \quad\left(\iota>\iota_{0} ; u<0\right)
\end{aligned}
$$

where the sum is over all roots $s_{r}$ of (10.3) which lie on the imaginary axis. The residue at a root $s_{r}$ of multiplicity $l_{r}+1$ has the form $e^{t_{r}} p_{l}(t)$, where $p_{l}(t)$ is a polynomial vector of degree at most $l_{r}$. These terms are bounded as $t \rightarrow+\infty$ if $l_{r}=0$ ( $s_{r}$ is a simple root), and also in any case in which $p_{l}(t)$ is constant. A sufficient condition for this is that the residue of $e^{t_{s}} T^{-1}(s)$ at $s_{r}$ be a constant multiple of $e^{t_{r}}$. When this is the case, we shall call $s_{r}$ a root of simple type of (10.3).

The integral in (10.9) has the form

$$
\frac{e^{t u}}{2 \pi} \int_{-\infty}^{+\infty} e^{i t v} T^{-1}(u+i v) y^{*}(u+i v) d v \quad(u<0) .
$$

Now from (10.5) we see that any cofactor in $\operatorname{det} T(s)$ has the form $\sum q_{j}(s) e^{s k j}$, where $k_{j}$ is a combination of the values $h_{n}$ and each $q_{j}(s)$ is a scalar polynomial of degree $N-1$ or less. Moreover, by integrating the first integral in (10.6) by parts, we find that

$$
\left.y^{*}(s)=y\left(t_{0}\right) e^{s\left(h_{m}-t_{0}\right.}\right)+e^{s h_{m}} \int_{t_{0}}^{t_{0}+h_{m}} y^{\prime}(t) e^{-s t} d t+\sum_{n=0}^{m} B_{n} e^{s h_{n}} \int_{t_{0}}^{t_{0}+h_{n}} y(t) e^{-s t} d t .
$$

Therefore, a typical term in an element in the matrix (10.10) is of the form

$$
e^{(t+c) u} \int_{-\infty}^{+\infty} e^{i(t+c) v}\{\operatorname{det} T(u+i v)\}^{-1}(u+i v)^{i} \int_{t_{0}}^{t_{0}+h_{n}} y_{1}\left(t_{1}\right) e^{-(u+i v) t_{1}} d t_{1} d v
$$

where $c$ is a constant, $j \leqq N-1$, and $y_{1}$ is an element of the vector $y$. Since

$$
\operatorname{det} T(u+i v)=e^{(u+i v) h_{m}}\left\{(u+i v)^{N}+O\left(|u+i v|^{N-1}\right)\right\}
$$

as $|u+i v| \rightarrow \infty$, the above integral has convergence properties analogous to those of

$$
e^{(t+c) u} \int_{-\infty}^{\infty} e^{i(t+c) v}(u+i v)^{-k} \int_{t_{0}}^{t_{0}+h_{n}} y_{1}\left(t_{1}\right) e^{-(u+i v) t_{1}} d t_{1} d v
$$

where $k \geqq 1$ and $c$ is a constant. If $k>1$, the infinite integral is absolutely 
convergent and bounded in $t$, and since $u<0$ the expression (10.11) tends to zero as $t \rightarrow+\infty$. If $k=1$, the infinite integral is boundedly convergent, and (10.11) tends to zero. Thus the integral in (10.9) is $o(1)$ as $t \rightarrow+\infty$, and the boundedness of $y(t)$ depends on the boundedness of the residue discussed in the preceding paragraph.

The above remarks prove the sufficiency of the following condition.

Theorem 6. A necessary and sufficient condition in order that all solutions of

$$
y^{\prime}\left(t+h_{m}\right)+\sum_{n=0}^{m} B_{n} y\left(t+h_{n}\right)=0,
$$

continuous for $t>t_{0}$, be bounded as $t \rightarrow+\infty$, is that all roots of the transcendental equation

$$
\operatorname{det} T(s) \equiv \operatorname{det}\left(s e^{s h_{m}} I+\sum_{n=0}^{m} B_{n} e^{s h_{n}}\right)=0
$$

have nonpositive real parts, and that all with zero real parts be of simple type. $A$ root $s_{r}$ is of simple type if the residue of $e^{t_{s}} T^{-1}(s)$ at $s_{r}$ is a constant multiple of $e^{t_{s}}$, and in particular if $s_{r}$ is a root of multiplicity one.

The necessity of this condition can be proved by the following argument. Let $s_{r}$ be any root with zero real parts, and let $y(t)$ be defined by the equation

$$
y(t)=\int e^{t_{s}} T^{-1}(s) d s,
$$$$
t>t_{0},
$$

where the integration is around a fixed circle which has its center at $s_{r}$ and which contains no other root of (10.3). Then it is easy to verify that the function in (10.13) is a solution of (10.12). From (10.13), we see that this solution is $2 \pi i$ times the residue at $s_{r}$ of $e^{t_{s}} T^{-1}(s)$. Thus if $s_{r}$ is not of simple type, (10.12) will possess an unbounded solution.

11. The scalar equation of neutral type: integral representation of solutions. We shall now turn to the more difficult problem of analyzing equations of neutral type such as the scalar equation

$$
u^{\prime}(t+h)+a(t) u^{\prime}(t)+b(t) u(t)+c(t) u(t+h)=w(t) .
$$

We shall first indicate how the continuation procedure can be used in proving the existence of solutions. An appropriate initial condition for (11.1) is again of the form

$$
u(t)=\phi(t), \quad t_{0} \leqq t \leqq t_{0}+h,
$$

where $\phi(t)$ is a given real function. Let us suppose that $a(t), b(t)$, and $c(t)$ are continuous for $t \geqq t_{0}$, that $\phi^{\prime}(t)$ is continuous for $t_{0}<t<t_{0}+h$, and that $w(t)$ is continuous for $t \geqq t_{0}$ except for possible jump discontinuities at the points 
$t_{0}+n h(n=0,1,2,3, \cdots)$. Then from (11.1), it follows that there is just one way to define $u(t)$ over $t_{0}+h<t<t_{0}+2 h$ so that $u(t)$ is continuous at $t_{0}+h$, equal to $\phi(t)$ for $t_{0} \leqq t \leqq t_{0}+h$, and so that (11.1) is satisfied for $t_{0}<t<t_{0}+h$. The derivative $u^{\prime}(t)$ will be defined and continuous for $t_{0}+h<t<t_{0}+2 h$, but will, in general, have a finite jump discontinuity at $t_{0}+h$. By repeating this argument, we can continue $u(t)$ from one interval of length $h$ to another, the continuation being made unique by requiring continuity of $u$. In contrast to the situation for equations with retarded argument, the discontinuity in the derivative of $u$ at $t_{0}+h$ can be propagated; that is, $u^{\prime}(t)$ may have jumps at the points $t_{0}+n h(n=1,2,3, \cdots)\left({ }^{14}\right)$. If $u^{\prime}(t)$ happens to be continuous at $t_{0}+h$, then it will be continuous for all $t>t_{0}$.

Definition. Any continuous function $u(t)$, determined in the above way by an initial function $\phi(t)$ with a continuous derivative, will be called a continuous solution of (11.1).

Note that (11.1) actually need be satisfied only for $t>t_{0}, t \neq t_{0}+n h$ $(n=1,2,3, \cdots)$. With this definition, we see that there is a unique continuous solution of (11.1) and (11.2).

The adjoint equation and kernel $v(s, t)$ are defined as follows, assuming continuous differentiability of $a(s)$.

Definition. Let $v(s, t)$ denote the unique function which satisfies the adjoint equation

$$
\begin{aligned}
-\frac{\partial v(s, t)}{\partial s}-\frac{\partial}{\partial s}[v(s+h, t) a(s & +h)] \\
& +v(s+h, t) b(s+h)+v(s, t) c(s)=0
\end{aligned}
$$

for $t_{0}<s<t, s \neq t-n h(n=0,1,2, \cdots)$, which satisfies the initial condition

$$
v(s, t)=\left\{\begin{array}{ll}
0, & t<s \leqq t+h \\
1, & s=t
\end{array}\right\}
$$

and which further satisfies the condition that

$$
v(s, t)+v(s+h, t) a(s+h)
$$

be a continuous function $\left({ }^{15}\right)$ of $s$ for $t_{0} \leqq s \leqq t$.

If we regard (11.3) as an equation for $s<t$, we see that it is of neutral type. The continuation process can be applied to extend the function $v(s, t)$ from the interval $t<s \leqq t+h$ back to the interval $t-h<s \leqq t$, and so forth, until finally it is defined over $t_{0} \leqq s \leqq t$. The continuation is made unique by the continuity condition (11.5). At the points $t, t-h, t-2 h, \cdots, v(s, t)$ can have finite jumps determined by the conditions (11.4) and (11.5), and of course $\partial v(s, t) / \partial s$ will also have jumps at these points. Elsewhere $v$ and $\partial v / \partial s$ will be

(14) This is true even if $w(t)$ is everywhere continuous.

(15) This should be understood to mean continuous on the right at $t_{0}$ and on the left at $t$. 
continuous. Once again, it is not essential to define $v(s, t)$ for $t<s \leqq t+h$, but it is more convenient to do so.

From Equation (11.1) we now obtain

$$
\begin{aligned}
\int_{t_{0}}^{t} v(s, t)\left[u^{\prime}(s+h)+a(s)\right. & \left.u^{\prime}(s)\right] d s+\int_{t_{0}}^{t} v(s, t) b(s) u(s) d s \\
& +\int_{t_{0}}^{t} v(s, t) c(s) u(s+h) d s=\int_{t_{0}}^{t} v(s, t) w(s) d s
\end{aligned}
$$

since each integrand is piecewise continuous. Now, since $u(s)$ and $v(s, t)$ $+a(s+h) v(s+h, t)$ have derivatives with only finite jump discontinuities, they are of bounded variation for $t_{0} \leqq s \leqq t$. Since both functions are continuous, this permits the integration by parts below. We use the assumption that $u(t)=0$ for $t_{0} \leqq t \leqq t_{0}+h$ and the fact that $v(s, t)=0$ for $t<s \leqq t+h$.

$$
\begin{aligned}
\int_{t_{0}}^{t} \frac{\partial}{\partial s}[v(s, t)+a(s & +h) v(s+h, t)] u(s+h) d s \\
& =u(t+h)-\int_{t_{0}}^{t}[v(s, t)+a(s+h) v(s+h, t)] u^{\prime}(s+h) d s \\
& =u(t+h)-\int_{t_{0}}^{t} v(s, t)\left[u^{\prime}(s+h)+a(s) u^{\prime}(s)\right] d s .
\end{aligned}
$$

Since, moreover,

$$
\int_{t_{0}}^{t} v(s, t) b(s) u(s) d s=\int_{t_{0}}^{t} v(s+h, t) b(s+h) u(s+h) d s,
$$

Equation (11.6) can be put in the form

$$
\begin{array}{rlr}
u(t+h)+\int_{t_{0}}^{t}\{ & -\frac{\partial}{\partial s}[v(s, t)+a(s+h) v(s+h, t)] \\
& +v(s+h, t) b(s+h)+v(s, t) c(s)\} u(s+h) d s & \\
& =\int_{t_{0}}^{t} v(s, t) w(s) d s, & \iota>t_{0} .
\end{array}
$$

Using (11.3), we finally obtain the representation

$$
u(t+h)=\int_{t_{0}}^{t} v(s, t) w(s) d s, \quad\left(t>t_{0}\right)
$$

12. The scalar equation of neutral type: representation of the derivative of a solution. As we shall see in $\$ 14$, we also need a representation for $u^{\prime}(t+h)$. 
In accordance with our assumptions, $w(s)$ is continuous for $t_{0} \leqq s \leqq t, s \neq t_{0}$ $+n h(n=1,2,3, \cdots)$, and $v(s, t)$ is continuous for $t_{0} \leqq s \leqq t, s \neq t-n h$ $(n=0,1,2, \cdots)$. Provided $t-t_{0}$ is not a multiple of $h$, the sets $\left\{t_{0}+n h\right\}$ and $\{t-n h\}$ interlace, and

$$
\begin{aligned}
u(t+h)= & \int_{t_{0}}^{t-N h} v(s, t) w(s) d s+\int_{t_{-N h}}^{t_{0}+h} v(s, t) w(s) d s \\
& +\int_{t_{0}+h}^{t-(N-1) h} v(s, t) w(s) d s+\cdots \\
& +\int_{t-h}^{t_{0}+N h} v(s, t) w(s) d s+\int_{t_{0}+N h}^{t} v(s, t) w(s) d s,
\end{aligned}
$$

where $N$ is a suitably chosen integer. Each integrand is now continuous, and differentiation yields

$$
\begin{aligned}
u^{\prime}(t+h)= & w(t)+\int_{t_{0}}^{t}\left\{\frac{\partial v}{\partial t}(s, t)\right\} w(s) d s \\
& -\sum_{n=1}^{N} w(t-n h)[v(t-n h+, t)-v(t-n h-, t)], \\
& \quad t>t_{0}, t \neq t_{0}+k h, k=1,2, \cdots .
\end{aligned}
$$

Existence and continuity of $\partial v(s, t) / \partial t$, needed in the preceding demonstration, can be established by the following device. For any $r \geqq t_{0}$, let $u(t, r)$ denote the unique function which satisfies the equation

$$
\Lambda u(t, r)=\frac{\partial u}{\partial t}(t+h, r)+a(t) \frac{\partial u}{\partial t}(t, r)+b(t) u(t, r)+c(t) u(t+h, r)=0,
$$

and the conditions

$$
t>r, t \neq r+n h, n=0,1,2, \cdots,
$$

$$
u(t, r)=\left\{\begin{array}{ll}
0, & r \leqq t<r+h \\
1, & t=r+h
\end{array}\right\},
$$

(iii) $\quad u(t+h, r)+a(t) u(t, r)$ is a continuous function of $t$ for $t \geqq r$.

The continuation process shows that the $u(t, r)$ exists, is unique, and that $u(t, r)$ and $\partial u(t, r) / \partial t$ are continuous for $t>r$ except for possible jumps at $t=r+n h(n=1,2,3, \cdots)$. Moreover, for any $z>r, z \neq r+n h$, let $v(t, z)$ have the meaning previously defined:

$$
\begin{aligned}
& \Lambda^{*} v(t, z)=-\frac{\partial v}{\partial t}(t, z)-\frac{\partial}{\partial t}[v(t+h, z) a(t+h)] \\
&+v(t+h, z) b(t+h)+v(t, z) c(t)=0, \\
& t_{0}<t<z, t \neq z-n h, n=0,1,2, \cdots,
\end{aligned}
$$




$$
v(t, z)=\left\{\begin{array}{ll}
0, & z<t \leqq z+h \\
1, & t=z
\end{array}\right\},
$$

(iii') $\quad v(t, z)+v(t+h, z) a(t+h)$ is a continuous function of $t$ for $t_{0} \leqq t \leqq z$. $v(t, z)$ and $\partial v(t, z) / \partial t$ are continuous for $t_{0}<t<z, t \neq z-n h(n=0,1,2, \cdots)$. Now consider the expression,

$$
\int_{r}^{z}\left[v(s, z) \Lambda u(s, r)-u(s+h, r) \Lambda^{*} v(s, z)\right] d s,
$$

which is seen to be zero, under the foregoing conditions. By (ii) and (ii'),

$$
\int_{r}^{z} u(s+h, r) v(s+h, z) b(s+h) d s=\int_{r}^{z} u(s, r) v(s, z) b(s) d s,
$$

and similarly

$$
\int_{r}^{z} u(s+h, r)[v(s+h, z) a(s+h)]^{\prime} d s=\int_{r}^{z} u(s, r)[v(s, z) a(s)]^{\prime} d s .
$$

Combining these results, we of tain

$$
\int_{r}^{z} \frac{d}{d s}\{v(s, z)[u(s+h, r)+a(s) u(s, r)]\} d s=0 .
$$

From (iii) and ( iii $^{\prime}$ ) we see that the integrand is continuous except at $s=z$, $z-h, \cdots, z-M h$, where $M$ is the largest integer for which $r<z-M h$. Hence we get

$$
\begin{gathered}
v(z, z)[u(z+h, r)+a(z) u(z, r)]-v(r, z)[u(r+h, r)+a(r) u(r, r)] \\
+\sum_{j=1}^{M}[v(z-j h-, z)-v(z-j h+, z)][u(z-j h+h, r) \\
\quad+a(z-j h) u(z-j h, r)]=0 .
\end{gathered}
$$

Using (ii) and $\left(\mathrm{ii}^{\prime}\right)$, this takes the form $\left({ }^{16}\right)$

$$
\begin{aligned}
v(r, z)= & u(z+h, r)+a(z) u(z, r)-\sum_{j=1}^{M}[v(z-j h+, z)-v(z-j h-, z)] \\
& \cdot[u(z-j h+h, r)+a(z-j h) u(z-j h, r)] .
\end{aligned}
$$

From (ii') and (iii') we readily find that

(16) A slight variation of this argument yields the reciprocal relation $u(z+h, r)=v(r, z)$ $+v(r+h, z) a(r+h)+\sum_{j=1}^{N}[v(r+j h, z)+v(r+j h+h, z) a(j h+h)][u(r+j h+h+, r)$ $-u(r+j h+h-, r)]$. 


$$
\begin{aligned}
& v(z-h+, z)-v(z-h-, z)=a(z), \\
& \begin{aligned}
v(z-j h+, z)-v(z-j h-, z) \\
=-a(z-j h+h)[v(z-j h+h+, z)-v(z-j h+h-, z)], \\
\quad j=2,3, \cdots, M .
\end{aligned}
\end{aligned}
$$

Thus

$$
\begin{array}{r}
v(z-j h+, z)-v(z-j h-, z)=(-1)^{i-1} a(z) a(z-h) \cdots a(z-j h+h), . \\
j=1,2, \cdots, M .
\end{array}
$$

Since $a(t)$ has a continuous derivative for $t>t_{0}$, it follows that $v(z-j h+, z)$ $-v(z-j h-, z)$ has a continuous derivative, with respect to $z$, for $z>r$, $z \neq r+n h, n=0,1,2, \cdots, j=1,2, \cdots, M$. Moreover, $\partial u(z, r) / \partial z$ exists and is continuous for $z>r, z \neq r+n h(n=0,1,2, \cdots)$. It is therefore clear from (12.2) that $\partial v(r, z) / \partial z$ exists and is continuous for $z>r \geqq t_{0}, z \neq r+n h$ $(n=0,1,2, \cdots)$. This is just the result needed in establishing (12.1).

The results of $\S \S 11$ and 12 are summarized in the following theorem.

THEOREM 7. Suppose that $b(t)$ and $c(t)$ are continuous and that $a(t)$ has a continuous derivative for $t \geqq t_{0}$, and suppose that $w(t)$ is continuous for $t \geqq t_{0}$ except for possible jump discontinuities at the points $t_{0}+n h(n=1,2,3, \cdots)$. Let $v(s, t)$ denote the solution of the adjoint equation defined above. Then the unique continuous solution $u(t)$ of

$$
\begin{gathered}
u^{\prime}(t+h)+a(t) u^{\prime}(t)+b(t) u(t)+c(t) u(t+h)=w(t), \\
t>t_{0}, t \neq t_{0}+n h, n=1,2, \cdots,
\end{gathered}
$$

subject to the initial condition $u(t)=0$ for $t_{0} \leqq t \leqq t_{0}+h$, is given by the formula

$$
u(t+h)=\int_{t_{0}}^{t} v(s, t) w(s) d s .
$$

Moreover, $\partial v(s, t) / \partial t$ exists and is continuous for $t>s \geqq t_{0}, t \neq s+n h$ $(n=0,1,2, \cdots)$, and

$$
\begin{aligned}
u^{\prime}(t+h)= & w(t)+\int_{t_{0}}^{t}\left\{\frac{\partial v}{\partial t}(s, t)\right\} w(s) d s \\
- & -\sum_{n=1}^{N} w(t-n h)[v(t-n h+, t)-v(t-n h-, t)], \\
& \quad t>t_{0}, t \neq t_{0}+k h, k=1,2, \cdots,
\end{aligned}
$$

where $N$ is the greatest integer such that $t-N h>t_{0}$.

In the next section, we shall state and prove the corresponding result for the general matrix system of equations of neutral type. 
13. Systems of equations of neutral type. As we have already remarked, the most general linear, nonhomogeneous system of differential-difference equations has the form (4.1). If $A_{m}(t)$ is nonsingular, for $t>t_{0}$, the equation is said to be of neutral type. In this case, we can multiply by $A_{m}^{-1}(t)$, and therefore we may as well consider the system

$$
z^{\prime}\left(t+h_{m}\right)+\sum_{n=0}^{m-1} A_{n}(t) z^{\prime}\left(t+h_{n}\right)+\sum_{n=0}^{m} B_{n}(t) z\left(t+h_{n}\right)=w(t), \quad t>t_{0} .
$$

The initial condition is again of the form

$$
z(t)=\phi(t), \quad t_{0} \leqq t \leqq t_{0}+h_{m},
$$

where $\phi(t)$ is a given vector. In order to facilitate the discussion of the continuation method, let us define the set $S$ to be the set of points of the form

$$
t_{0}+j h_{m}-i_{1} h_{1}-i_{2} h_{2}-\cdots-i_{m-1} h_{m-1}
$$

where $j, i_{1}, \cdots, i_{m-1}$ are integers, $j=1,2,3, \cdots$, and $0 \leqq i_{1}+i_{2}+\cdots$ $+i_{m-1} \leqq j$. Let $S^{\prime}$ denote the subset of $S$ consisting of points (13.3) for which $0 \leqq i_{1}+i_{2}+\cdots+i_{m-1} \leqq j-1$. The sets $S$ and $S^{\prime}$ have no finite limit points, since

$$
\begin{aligned}
j h_{m}-i_{1} h_{1}-\cdots-i_{m-1} h_{m-1} & \geqq j h_{m}-\left(i_{1}+i_{2}+\cdots+i_{m-1}\right) h_{m-1} \\
& >j\left(h_{m}-h_{m-1}\right)
\end{aligned}
$$

and the latter expression tends to infinity with $j$. It follows that the points of $S$ divide the interval $\left(t_{0},+\infty\right)$ into a countable set of open intervals. Let the points of $S$ be linearly ordered and labelled $t_{1}, t_{2}, \cdots$, where $t_{0}<t_{1}$ $<t_{2}<\cdots$.

Now let us suppose that each $A_{n}(t)$ and each $B_{n}(t)$ is continuous for $t \geqq t_{0}$, that $\phi^{\prime}(t)$ is continuous for $t_{0}<t<t_{0}+h_{m}$, and that $w(t)$ is continuous for $t \geqq t_{0}$ except for possible jump discontinuities on the set $S$. Then from (13.1) we see that $z^{\prime}\left(t+h_{m}\right)+B_{m}(t) z\left(t+h_{m}\right)$ is determined over $t_{0}<t<t_{0}$ $+h_{m}-h_{m-1}$, by the given $\phi(t)$, as a continuous vector function. Thus there exists a unique continuation of $\phi(t)$ to a continuous function $z(t)$ defined for $t_{0} \leqq t \leqq t_{0}+2 h_{m}-h_{m-1}=t_{1}+h_{m}$. The derivative $z^{\prime}(t)$ is continuous in this interval except at $t_{0}+h_{m}$, and Equation (13.1) is satisfied for $t_{0}<t<t_{0}+h_{m}-h_{m-1}$ $=t_{1}$. Repeating this argument, we see that $z^{\prime}\left(t+h_{m}\right)+B_{m}(t) z\left(t+h_{m}\right)$ is determined by (13.1) for $t_{0}+h_{m}-h_{m-1}=t_{1}<t<\min \left(t_{0}+2 h_{m}-2 h_{m-1}, t_{0}+h_{m}-h_{m-2}\right)$. Thus $z(t)$ is uniquely continuable as a continuous function to $t_{0} \leqq t \leqq t_{2}+h_{m}$. Its derivative $z^{\prime}(t)$ is continuous in this interval except at points of $S^{\prime}$, and (13.1) is satisfied except at points of $S$. By repeating this procedure, we find that there is a unique continuous vector $z(t)$ which satisfies (13.1) for $t>t_{0}, t \notin S$ and which satisfies (13.2). $z^{\prime}(t)$ is continuous for $t>t_{0}, t \in S^{\prime}$.

The adjoint system for (13.1) is 


$$
\begin{aligned}
-\frac{\partial Y}{\partial s}(s, t) & -\sum_{n=0}^{m-1} \frac{\partial}{\partial s}\left[Y\left(s+h_{m}-h_{n}, t\right) A_{n}\left(s+h_{m}-h_{n}\right)\right] \\
& +\sum_{n=0}^{m} Y\left(s+h_{m}-h_{n}, t\right) B_{n}\left(s+h_{m}-h_{n}\right)=0
\end{aligned}
$$

assuming that each $A_{n}$ has a continuous derivative. This equation is itself of neutral type, and associated with it are sets, similar to $S$ and $S^{\prime}$, which we shall call $T$ and $T^{\prime} . T$ is defined as the set of points of the form

$$
t+h_{m}-j h_{m}+i_{1} h_{1}+\cdots+i_{m-1} h_{m-1}
$$

where $j=1,2,3, \cdots, 0 \leqq i_{1}+i_{2}+\cdots+i_{m-1} \leqq j . T^{\prime}$ is the subset of $T$ for which $0 \leqq i_{1}+i_{2}+\cdots+i_{m-1} \leqq j-1$. It is not difficult to show, from an argument similar to that given above, that there is a unique continuous matrix $Y(s, t)$ which satisfies (13.4) for $t_{0}<s<t, s \in T$, and which has prescribed continuously differentiable initial values for $t \leqq s \leqq t+h_{m}$. Indeed, a slight modification of the previous argument can be used to prove the existence and uniqueness of the kernel function defined as follows.

DEFINITION. Let $Y(s, t)$ denote the unique matrix function which satisfies the adjoint system (13.4) for $t_{0}<s<t, s \in T$, which satisfies the initial condition

$$
Y(s, t)=\left\{\begin{array}{ll}
0, & t<s \leqq t+h_{m} \\
I, & s=t
\end{array}\right\},
$$

and which satisfies the requirement that

$$
Y(s, t)+\sum_{n=0}^{m-1} Y\left(s+h_{m}-h_{n}, t\right) A_{n}\left(s+h_{m}-h_{n}\right)
$$

be a continuous function $\left.{ }^{17}\right)$ of $s$ for $t_{0} \leqq s \leqq t$.

Moreover, the argument shows that $\partial Y(s, t) / \partial s$ is continuous for $t_{0}<s<t$ $+h_{m}, s \notin T^{\prime} . Y(s, t)$ itself is continuous for $t_{0} \leqq s \leqq t+h_{m}, s \notin T^{\prime}$.

We can now obtain the desired representation formula for the solution of the inhomogeneous equation just as before. From Equation (13.1) we get

$$
\begin{aligned}
\int_{t_{0}}^{t} Y(s, t) z^{\prime}\left(s+h_{m}\right) d s & +\sum_{n=0}^{m-1} \int_{t_{0}}^{t} Y(s, t) A_{n}(s) z^{\prime}\left(s+h_{n}\right) d s \\
& +\sum_{n=0}^{m} \int_{t_{0}}^{t} Y(s, t) B_{n}(s) z\left(s+h_{n}\right) d s \\
& =\int_{t_{0}}^{t} Y(s, t) w(s) d s .
\end{aligned}
$$

Since $z\left(s+h_{m}\right)$ and the function in (13.7) are continuous for $t_{0} \leqq s \leqq t$, and

(17) Continuous on the right at $t_{0}$ and on the left at $t$. 
have derivatives with only finite jump discontinuities, we can integrate

$$
\int_{t_{0}}^{t} \frac{\partial}{\partial s}\left\{Y(s, t)+\sum_{n=0}^{m-1} Y\left(s+h_{m}-h_{n}, t\right) A_{n}\left(s+h_{m}-h_{n}\right)\right\} z\left(s+h_{m}\right) d s
$$

by parts. Assuming that $z(t)=0$ for $t_{0} \leqq t \leqq t_{0}+h_{m}$, and recalling (13.6), we obtain in this way

$z\left(t+h_{m}\right)-\int_{t_{0}}^{t}\left\{Y(s, t)+\sum_{n=0}^{m-1} Y\left(s+h_{m}-h_{n}, t\right) A_{n}\left(s+h_{n}-h_{n}\right)\right\} z^{\prime}\left(s+h_{m}\right) d s$.

Using a translation of the variable in the terms of the summation in this expression, and also in the second summation in (13.8), we can write Equation (13.8) in the form

$$
\begin{aligned}
z\left(t+h_{m}\right)+\int_{t_{0}}^{t}[ & -\frac{\partial}{\partial s}\left\{Y(s, t)+\sum_{n=0}^{m-1} Y\left(s+h_{m}-h_{n}, t\right) A_{n}\left(s+h_{m}-h_{n}\right)\right\} \\
& \left.+\sum_{n=0}^{m} Y\left(s+h_{m}-h_{n}, t\right) B_{n}\left(s+h_{m}-h_{n}\right)\right] z\left(s+h_{m}\right) d s \\
= & \int_{t_{0}}^{t} Y(s, t) w(s) d s,
\end{aligned}
$$

Using (13.4), we finally obtain the formula

$$
z\left(t+h_{m}\right)=\int_{t_{0}}^{t} Y(s, t) w(s) d s, \quad t>t_{0} .
$$

We can also obtain a representation formula for $z^{\prime}\left(t+h_{m}\right)$. We know that $w(s)$ is continuous for $t_{0} \leqq s \leqq t, s \notin S$, and that $Y(s, t)$ is continuous for $t_{0} \leqq s \leqq t, s \notin T^{\prime}$. Suppose that $t \notin S$, so that the points of $T^{\prime}$ are never points of $S$. The sets of points $S \cup T^{\prime}$ then divides the interval $\left(t_{0}, t\right)$ into a finite number of subintervals within each of which the integrand in (13.9) is continuous. Write (13.9) as the sum of integrals over these subintervals. Each of the points of $T^{\prime}$ is dependent on $t$, and appears as the upper limit of one integral and the lower limit of another (except $t$ itself, which appears only as an upper limit). The points of $S$, which appear as limits on the integrals, are not dependent on $t$. Therefore differentiation of (13.9) yields

$$
\begin{aligned}
z^{\prime}\left(t+h_{m}\right)= & w(t)+\int_{t_{0}}^{t}\left\{\frac{\partial}{\partial t} Y(s, t)\right\} w(s) d s \\
& -\sum_{s \in T^{\prime}-(t)}[Y(s+, t)-Y(s-, t)] w(s), \quad t>t_{0}, t \notin S,
\end{aligned}
$$

where $T^{\prime}-(t)$ denotes the set $T^{\prime}$ with the point $t$ removed. Existence and continuity of $\partial Y(s, t) / \partial t$ can be proved by an argument similar to that used in the scalar case. We have thus proved the following: 
Theorem 8. Suppose that each $B_{n}(t)$ is continuous and each $A_{n}(t)$ has a continuous derivative for $t \geqq t_{0}$, and that $w(t)$ is continuous for $t \geqq t_{0}$ except for possible jump discontinuities on the set $S$ consisting of all points

$$
\begin{aligned}
t_{0}+j h_{m}- & i_{1} h_{1}-\cdots-i_{m-1} h_{m-1}, \\
& \left(j=1,2,3, \cdots ; 0 \leqq i_{1}+\cdots+i_{m-1} \leqq j\right) .
\end{aligned}
$$

Let $Y(s, t)$ denote the kernel matrix defined above. Then the unique continuous vector function $z(t)$ which satisfies (13.1) for $t>t_{0}, t \notin S$, subject to the initial condition $z(t)=0$ for $t_{0} \leqq t \leqq t_{0}+h_{m}$, is given by the formula

$$
z\left(t+h_{m}\right)=\int_{t_{0}}^{t} Y(s, t) w(s) d s,
$$

Moreover,

$$
\begin{aligned}
z^{\prime}\left(t+h_{m}\right)= & w(t)+\int_{t_{0}}^{t}\left\{\frac{\partial}{\partial t} Y(s, t)\right\} w(s) d s \\
& -\sum_{s \in T^{\prime}-(t)}[Y(s+, t)-Y(s-, t)] w(s), \quad t>t_{0}, t \notin S,
\end{aligned}
$$

where $T^{\prime}$ denotes the set of points

$$
\begin{aligned}
& t+h_{m}-j h_{m}+i_{1} h_{1}+\cdots+i_{m-1} h_{m-1}, \\
& \quad j=1,2,3, \cdots ; 0 \leqq i_{1}+\cdots+i_{m-1} \leqq j-1 .
\end{aligned}
$$

14. Stability theorems for equations of neutral type. We shall now establish stability theorems, analogous to Theorems 3, 4, and 5, for equations of neutral type. In order to keep to a minimum the lengthy calculations required, we shall do this in detail only for the scalar equation (11.1), and merely state the principal results for the general system (13.1). Let us first introduce the following definition.

Definition. A continuous solution of (11.1) is said to be bounded as $t \rightarrow+\infty$ provided there is a constant $c_{1}$ such that

$$
|u(t)|+\left|u^{\prime}(t)\right| \leqq c_{1}, \quad t>t_{0}, \quad t \neq t_{0}+n h, \quad n=1,2, \cdots .
$$

We shall now prove the following:

TheOREM 9. Let $a(t), a_{1}(t), b(t), b_{1}(t), c(t)$, and $c_{1}(t)$ be continuous for $t \geqq t_{0}$, and let $a(t)$ have a continuous derivative for $t \geqq t_{0}$. Then a sufficient condition in order that all continuous solutions $\left({ }^{18}\right)$ of

$$
\begin{gathered}
u^{\prime}(t+h)+\left[a(t)+a_{1}(t)\right] u^{\prime}(t)+\left[b(t)+b_{1}(t)\right] u(t) \\
+\left[c(t)+c_{1}(t)\right] u(t+h)=0
\end{gathered}
$$

(18) As defined in $\$ 11$. 
be bounded as $t \rightarrow+\infty$ is that the following four requirements be met:

(i) all continuous solutions of

$$
u^{\prime}(t+h)+a(t) u^{\prime}(t)+b(t) u(t)+c(t) u(t+h)=0
$$

are bounded as $t \rightarrow+\infty$;

$$
\begin{gathered}
a_{1}(t), b_{1}(t), c_{1}(t) \rightarrow 0 \text { as } t \rightarrow \infty ; \\
\int^{\infty}\left|a_{1}(t)\right| d t, \quad \int \infty\left|b_{1}(t)\right| d t, \quad \int \infty\left|c_{1}(t)\right| d t<\infty ; \\
|v(s, t)| \leqq c_{2}, \quad\left|\frac{\partial v}{\partial t}(s, t)\right| \leqq c_{2},
\end{gathered}
$$

for $t>t_{0}, t_{0} \leqq s \leqq t, t-t_{0} \neq n h(n=0,1,2, \cdots)$. Here $v(s, t)$ is the kernel function for Equation (14.2), as defined in $\$ 12$.

Proof. From Equation (11.7), we know that every continuous solution of the nonhomogeneous equation (11.1), with $w(t)$ continuous except for jumps at $t_{0}+n h(n=1,2,3, \cdots)$, has the form

$$
u(t+h)=r(t+h)+\int_{t_{0}}^{t} v(s, t) w(s) d s
$$

where $r(t)$ is a continuous solution of the corresponding homogeneous equation (14.2). Equation (14.1) has the form of (11.1) if we write

$$
w(t)=-a_{1}(t) u^{\prime}(t)-b_{1}(t) u(t)-c_{1}(t) u(t+h) .
$$

For a continuous solution $u$ of $(14.1)$, we know from $\S 11$ that $u^{\prime}(t)$ is continuous except at $t=t_{0}+n h(n=1,2,3, \cdots)$; hence $w(t)$ is continuous except at these points. Consequently a continuous solution $u$ of (14.1) must satisfy the integral equation

$$
u(t+h)=r(t+h)-\int_{t_{0}}^{t} v(s, t)\left[a_{1}(s) u^{\prime}(s)+b_{1}(s) u(s)+c_{1}(s) u(s+h)\right] d s .
$$

Furthermore, from Equation (12.1),

$$
\begin{aligned}
u^{\prime}(t+h)= & -a_{1}(t) u^{\prime}(t)-b_{1}(t) u(t)-c_{1}(t) u(t+h)+r^{\prime}(t+h) \\
& -\int_{t_{0}}^{t}\left\{\frac{\partial}{\partial t} v(s, t)\right\}\left[a_{1}(s) u^{\prime}(s)+b_{1}(s) u(s)+c_{1}(s) u(s+h)\right] d s \\
& +\sum_{n=1}^{N}\left[a_{1}(t-n h) u^{\prime}(t-n h)+b_{1}(t-n h) u(t-n h)\right. \\
& \left.+c_{1}(t-n h) u(t-n h+h)\right][v(t-n h+, t)-v(t-n h-, t)],
\end{aligned}
$$

$t>t_{0}, t \neq t_{0}+k h, k=1,2, \cdots$, where $N$ is the greatest integer such that $t-N h>t_{0}$.

By hypothesis (iv), 


$$
\begin{aligned}
|u(t+h)| \leqq & |r(t+h)|+c_{1} \int_{t_{0}}^{t}\left|a_{1}(s) u^{\prime}(s)+b_{1}(s) u(s)+c_{1}(s) u(s+h)\right| d s \\
\left|u^{\prime}(t+h)\right| \leqq & \left|r^{\prime}(t+h)\right|+\left|a_{1}(t) u^{\prime}(t)+b_{1}(t) u(t)+c_{1}(t) u(t+h)\right| \\
& +c_{1} \int_{t_{0}}^{t}\left|a_{1}(s) u^{\prime}(s)+b_{1}(s) u(s)+c_{1}(s) u(s+h)\right| d s \\
& +2 c_{1} \sum_{n=1}^{N} \mid a_{1}(t-n h) u^{\prime}(t-n h)+b_{1}(t-n h) u(t-n h) \\
& \quad+c_{1}(t-n h) u(t-n h+h) \mid
\end{aligned}
$$

$t>t_{0}, t \neq t_{0}+k h, k=1,2, \cdots$. By hypothesis, there exists a function $g(t)$, which decreases monotonically to zero as $t \rightarrow+\infty$, which satisfies the condition

$$
g(t) \geqq \max \left(\left|a_{1}(t)\right|,\left|b_{1}(t)\right|,\left|c_{1}(t-h)\right|,\left|c_{1}(t)\right|\right)
$$

for all $t \geqq t_{0}$, and for which $\int_{t_{0}}^{\infty} g(s) d s<\infty$. Then, taking into account the boundedness of $r$ and $r^{\prime}$, (hypothesis i), we have

$$
\begin{aligned}
|u(t+h)| \leqq & c_{1}+c_{2} \int_{t_{0}}^{t} g(s)\left(|u(s)|+\left|a^{\prime}(s)\right|\right) d s \\
& \quad+c_{2} \int_{t_{0}}^{t+h} g(s)|u(s)| d s, \quad t> \\
\left|u^{\prime}(t+h)\right| \leqq & c_{1}+g(t)\left(|u(t)|+\left|u^{\prime}(t)\right|\right)+g(t)|u(t+h)| \\
& +c_{2} \int_{t_{0}}^{t} g(s)\left(|u(s)|+\left|u^{\prime}(s)\right|\right) d s \\
& +c_{2} \int_{t_{0}}^{t+h} g(s)|u(s)| d s \\
& +4 c_{2} \sum_{n=0}^{N} g(t-n h)\left(\left|u^{\prime}(t-n h)\right|+|u(t-n h)|\right),
\end{aligned}
$$

$t>t_{0} ; t \neq t_{0}+k h, k=1,2, \cdots$. Let

$$
\|u(t)\|=\left|u^{\prime}(t)\right|+|u(t)|
$$

and take $t_{0}$ sufficiently large $\left({ }^{19}\right)$. Then the expressions given above yield

$$
\|u(t+h)\|_{1} \leqq c_{3}+c_{3} \int_{t_{0}}^{t+h} g(s)\|u(s)\| d s+c_{3} \sum_{n=0}^{N} g(t-n h)\|u(t-n h)\| .
$$

(19) It is permissible to suppose $t_{0}$ as large as required. For, given $t_{0}$, we can regard $u$ as determined from (14.1) by its values over any interval $\left(t_{0}+p h, t_{0}+p h+h\right)$ where $p$ is any positive integer. 
Let

$$
u_{1}(t)=\max _{t_{0} \leqq s \leqq t}\|u(s)\|
$$

Then $u_{1}(t)$ is monotone increasing. It follows that

$$
\begin{aligned}
\sum_{n=0}^{N} g(t-n h)\|u(t-n h)\| & \leqq \sum_{n=0}^{N} g(t-n h) u_{1}(t-n h) \\
& \leqq \frac{1}{h} \sum_{n=0}^{N} \int_{t-n h}^{t-(n-1) h} g(r-h) u_{1}(r) d r
\end{aligned}
$$

Hence

$$
\|u(t+h)\| \leqq c_{3}+c_{3} \int_{t_{0}}^{t+h} g(s) u_{1}(s) d s+\frac{c_{3}}{h} \int_{t_{0}}^{t+h} g(r-h) u_{1}(r) d r .
$$

Thus

$$
u_{1}(t+h) \leqq c_{4}+c_{4} \int_{t_{0}}^{t+h} g(s-h) u_{1}(s) d s
$$

which yields

$$
u_{1}(t+h) \leqq c_{4} \exp \left[c_{4} \int_{t_{0}}^{t+h} g(s-h) d s\right]
$$

Thus $u_{1}(t)$ is bounded, and the proof of Theorem 9 is complete.

15. Stability theorems for equations of neutral type with constant coefficients. We shall conclude this paper with several theorems concerning stability of equations of neutral type with constant coefficients.

THEOREM 10. Let $a, b$, and $c$ be constants, let $a(t), b(t)$, and $c(t)$ be continuous for $t \geqq t_{0}$, and let $a(t)$ have a continuous derivative for $t \geqq t_{0}$. Then a sufficient condition in order that all continuous solutions of

$(15.1) u^{\prime}(l+h)+[a+a(l)] u^{\prime}(t)+[b+b(l)] u(t)+[c+c(t)] u(t+h)=0$

be bounded as $t \rightarrow+\infty$ is that the following three requirements be met:

(i) all solutions of

$$
u^{\prime}(t+h)+a u^{\prime}(t)+b u(t)+c u(t+h)=0
$$

for which $u(t+h)+$ au $(t)$ is continuous, are bounded as $t \rightarrow+\infty$;

(ii) $a(t), b(t)$, and $c(t)$ approach zero as $t \rightarrow+\infty$;

(iii) $\int \infty|a(t)| d t, \int \infty|b(t)| d t, \int \infty|c(t)| d t<\infty$.

Proof. We see from the adjoint equation (11.3) that $v(s, t)$ can be continued indefinitely in the negative $s$ direction, and hence can be regarded as 
defined for $-\infty<s \leqq t$. Make the substitutions

$$
\tau=t-s+t_{0}+h, \quad q(\tau, t)=v(s, t) .
$$

We see that $q(\tau, t)$ is defined for $t>t_{0}, t_{0} \leqq \tau<+\infty$, and satisfies

$$
\frac{\partial}{\partial \tau} q(\tau, t)+a \frac{\partial}{\partial \tau}[q(\tau-h, t)]+b q(\tau-h, t)+c q(\tau, t)=0
$$

for $t_{0}+h<\tau, \tau \neq t_{0}+n h(n=1,2,3, \cdots)$. Also

and

$$
q(\tau, t)= \begin{cases}0, & t_{0} \leqq \tau<t_{0}+h \\ 1, & \tau=t_{0}+h\end{cases}
$$

$$
q(\tau, t)+a q(\tau-h, t)
$$

is a continuous function of $\tau$ for $\tau \geqq t_{0}+h$. It is clear that $q$ is actually independent of $t$; we shall henceforth write $q(\tau)$ rather than $q(\tau, t)$. Condition (iv) of Theorem 9 can evidently be replaced by the condition that $\|q(\tau)\| \leqq c$ for $\tau>t_{0}$. However, $q(t)$ is a solution of (15.2), and $\|q(t)\| \leqq c$ is implied by condition (i) of Theorem 10. Since (i) of Theorem 10 also implies (i) of Theorem 9, we see now that Theorem 10 follows from Theorem 9 .

Similar theorems can be proved for systems of equations of neutral type. As we have already remarked, we shall omit the proofs of these theorems.

TheOREM 11. Let $A_{n}(t), B_{n}(t), C_{n}(t)$, and $D_{n}(t)$ be continuous for $t \geqq t_{0}$, and let $A_{n}(t)$ and $C_{n}(t)$ have continuous first derivatives for $t \geqq t_{0}$. Then a sufficient condition in order that all continuous solutions of the system

$$
\begin{aligned}
z^{\prime}\left(t+h_{m}\right) & +\sum_{n=0}^{m-1}\left[A_{n}(t)+C_{n}(t)\right] z^{\prime}\left(t+h_{m}\right) \\
& +\sum_{n=0}^{m}\left[B_{n}(t)+D_{n}(t)\right] z\left(t+h_{m}\right)=0, \quad t>t_{0}, t \in S,
\end{aligned}
$$

be bounded $\left({ }^{20}\right)$ as $t \rightarrow+\infty$ is that the following four requirements be met:

(i) all continuous solutions of

$$
z^{\prime}\left(t+h_{m}\right)+\sum_{n=0}^{m-1} A_{n}(t) z^{\prime}\left(t+h_{n}\right)+\sum_{n=0}^{m} B_{n}(t) z\left(t+h_{n}\right)=0, \quad t>\iota_{0}, \iota \notin S
$$

are bounded as $t \rightarrow+\infty$;

(ii) $C_{n}(t), D_{n}(t)$ tend to zero as $t \rightarrow+\infty$;

(iii) $\int \infty\left\|C_{n}(t)\right\| d t, \int \infty\left\|D_{n}(t)\right\| d t<\infty$;

(iv) $Y(s, t)$ is bounded for $t>t_{0}$;

${ }^{(20)}$ A solution of (15.3) is said to be bounded as $t \rightarrow+\infty$ if there is a constant $c_{1}$ such that $\|z(t)\|+\left\|z^{\prime}(t)\right\| \leqq c_{1}, t \in S^{\prime}, t>t_{0}$. 
$t_{0} \leqq s \leqq t, t \notin S$. Here $Y(s, t)$ denotes the kernel function for Equation (15.4), as defined in $\$ 13$.

Theorem 12. Let $A_{n}$ and $B_{n}$ be constants, let $C_{n}(t)$ and $D_{n}(t)$ be continuous for $t \geqq t_{0}$, and let $C_{n}(t)$ have a continuous derivative for $t \geqq t_{0}$. Suppose that the $A_{n}$ and $B_{n}$ commute: that is, each $A_{n}$ commutes with every $A_{k}$ and every $B_{l}$ and each $B_{n}$ commutes with every $A_{k}$ and every $B_{l}$. Then a sufficient condition in order that all continuous solutions of

$$
\begin{aligned}
z^{\prime}\left(t+h_{m}\right) & +\sum_{n=0}^{m-1}\left[A_{n}+C_{n}(t)\right] z^{\prime}\left(t+h_{n}\right) \\
& +\sum_{n=0}^{m}\left[B_{n}+D_{n}(t)\right] z\left(t+h_{n}\right)=0, \quad t>t_{0} ; t \in S,
\end{aligned}
$$

be bounded as $t \rightarrow+\infty$ is that the following three requirements be met:

(i) all solutions of

$$
z^{\prime}\left(t+h_{m}\right)+\sum_{n=0}^{m-1} A_{n} z^{\prime}\left(t+h_{n}\right)+\sum_{n=0}^{m} B_{n} z\left(t+h_{n}\right)=0, \quad t>t_{0}, t \notin S,
$$

for which $z\left(t+h_{m}\right)+\sum_{n=0}^{m-1} A_{n} z\left(t+h_{n}\right)$ is continuous, are bounded as $t \rightarrow+\infty$;

(ii) $\left\|C_{n}(t)\right\|$ and $\left\|D_{n}(t)\right\|$ tend to zero as $t \rightarrow+\infty$;

(iii) $\int \infty\left\|C_{n}(t)\right\| d t, \int \infty\left\|D_{n}(t)\right\| d t<\infty$.

\section{REFERENCES}

1. R. Bellman, Stability theory of differential equations, New York, McGraw-Hill Book Company, Inc., 1953.

2. R. Bellman and J. M. Danskin, A survey of the mathematical theory of time-lag, retarded control, and hereditary processes, The RAND Corporation, Report R-256, March 1, 1954.

3. N. G. de Bruijn, The difference-differential equation $F^{\prime}(x)=F(x-1) \exp (\alpha x+\beta)$, I and II, Indag. Math. vol. 15 (1953) pp. 449-464.

4. E. A. Coddington and N. Levinson, Theory of ordinary differential equations, New York, McGraw-Hill Book Company, Inc., 1955.

5. R. E. Langer, The asymptotic location of the roots of a certain transcendental equation, Trans. Amer. Math. Soc. vol. 31 (1929) pp. 837-844.

6. E. M. Wright, Linear difference-differential equations, Proc. Cambridge Philos. Soc. vol. 44 (1948) pp. 179-185.

7. - The linear difference-differential equation with constant coefficients, Proc. Roy. Soc. Edinburgh, Sect. A vol. 62 (1949) pp. 387-393.

8. - The stability of solutions of non-linear difference-differential equations, Proc. Roy. Soc. Edinburgh, Sect. A vol. 63 (1950) pp. 18-26.

The Rand Corporation, Santa Monica, Calif.

Pomona College, Claremont, Calif. 